\title{
Synthesis of novel bis[(tris(dimethylsilyl)methyl)alkyl]ferrocene derivatives as new ferrocenyl multi-functional silyl ether compounds
}

\author{
Reza Teimuri-Mofrad,* Hassan Abbasi, Kazem D. Safa, and Behnam Tahmasebi \\ Department of Organic and Biochemistry, Faculty of Chemistry, University of Tabriz, P.O. Box \\ 51666-16471, Tabriz, Iran \\ E-mail: teymouri@tabrizu.ac.ir
}

DOI: $\underline{\text { http://dx.doi.org/10.3998/ark.5550190.p009.596 }}$

\begin{abstract}
Some new branched polysilyl ethers with a ferrocene core were synthesized through treatment of 1,1'-bis[tris(dimethylsilyl)methyl]alkylferrocenes with various alcohols by Karstedt catalyst. Bis(chloroalkyl)ferrocenes are synthesized by acylation of ferrocene with 3-chloropropanoyl chloride and 4-chlorobutanoyl chloride. The obtained 1,1'-bis(3-chloropropanoyl)ferrocene and 1,1'-bis(4-chlorobutanoyl)ferrocene underwent a reduction and chlorides were replaced by bromides, and then they were treated with $\left(\mathrm{Me}_{3} \mathrm{Si}\right)_{3} \mathrm{CLi}$ and $\left(\mathrm{HMe}_{2} \mathrm{Si}\right)_{3} \mathrm{CLi}$ give silane compounds 6a-b and $7 \mathbf{a}-\mathbf{b}$.
\end{abstract}

Keywords: Ferrocene, silane, silyl ether, alcoholysis, dehydrocoupling

\section{Introduction}

Dendrimers with refined inner and outer structural conformations give access to macromolecular materials having special properties such as nanoscale catalysts, drug delivery, chemical sensors and so on. ${ }^{1-4}$ There are many publications which are concerned with the synthesis and identification of the dendritic compounds. ${ }^{5-6}$ The first dendrimers studied were those with an organic skeleton, and there were followed by transition metal-containing dendritic molecules. More recently, examples of dendrimers with silicon-included, such as carbosilane and siloxane, that have come to the fore..$^{7.8}$ The dendrimers including silicon can be classified into three main categories, having siloxane $(\mathrm{Si}-\mathrm{O}),{ }^{9}$ carbosilane $(\mathrm{Si}-\mathrm{C})^{10-15}$ or polysilane $(\mathrm{Si}-\mathrm{Si})^{16}$ backbones. Synthetic methods for the preparation of silicon-containing dendrimers were reported by many researchers who used the simple repeating procedures such as hydrosilylation, dehydrocoupling, alkenylation as well as alcoholysis. ${ }^{17-22}$

The chemistry of ferrocene-based structures has attracted much attention because of its importance in the fields of redox electrochemistry, materials science, novel materials, organic 
synthesis, ${ }^{23-26}$ catalysis for asymmetric synthesis, ${ }^{27-30}$ biology ${ }^{31-33}$ and non-linear optical materials. ${ }^{34-36}$ Recent discoveries of a broad range of applications in the biomedical and aerospace industries such as coating and catalysts greatly increaseds the interest in the incorporation of transition metals into organic monomers and polymers. ${ }^{37-41}$

Since the synthesis of the first silyl metal complex $\left(\eta^{5}-\mathrm{C}_{5} \mathrm{H}_{5}\right) \mathrm{Fe}(\mathrm{CO})_{2} \mathrm{SiMe}_{3}$ by Wilkinson in 1956 , research on transition metal silyl compounds has continued because these compounds are assumed to be the key intermediates in a number of important stoichiometric and catalytic transformations. ${ }^{42-43}$ Herein we report the first simple silyl etheric dendrimer generated with a ferrocene core and six branched silyl ethers by the dehydrocoupling reaction using the Karstedt catalyst.

\section{Results and Discussion}

We have recently used dehydrocoupling reactions for the preparation of tris(alkoxydimethylsilyl)methanes, ${ }^{44}$ calix[4] arenes bearing silylether groups ${ }^{45}$ and functionalized poly(methylalkoxy)siloxanes ${ }^{46}$ under mild conditions using Karstedt catalysis. We also applied this methodology in the synthesis of ferrocenyl silyl ethers for the attachment of ferrocenyl groups to the cellulose acetate butyrate backbone. ${ }^{47}$ In continuation of our work on ferrocene and silane compounds, we designed and synthesized some six new branched Si-H groups cored with a metallocene group. By applying our experiences from these methodologies, we aimed to introduce a completely new branched polysilyl ether with a ferrocene core.

1,1'-Bis(3- chloropropanoyl)ferrocene $\mathbf{3 a}$ and 1,1'-bis(4-chlorobutanoyl)ferrocene $\mathbf{3 b}$ were prepared by Friedel-Crafts acylation of ferrocene 1 with 3-chloropropanoyl chloride 2a and 4chlorobutanoyl chloride $\mathbf{2 b}$, respectively, in dry $\mathrm{CH}_{2} \mathrm{Cl}_{2}$ in the presence of $\mathrm{AlCl}_{3}$ as catalyst under reflux conditions. After reduction of the carbonyl groups in $\mathbf{3 a - b}$ by $\mathrm{NaBH}_{4}$ in diglyme, bis(chloroalkyl)ferrocene derivatives (4a-b) were obtained in high yields. ${ }^{48}$ The chlorine atoms in compunds 4a-b were replaced by bromine using ethyl bromide and sodium bromide in NMP $(\mathrm{N}$ methyl-2-pyrrolidone) as solvent at $60{ }^{\circ} \mathrm{C}$ for five days to give bis(bromoalkyl)ferrocene derivatives (5a-b) (Scheme 1). ${ }^{49}$ 


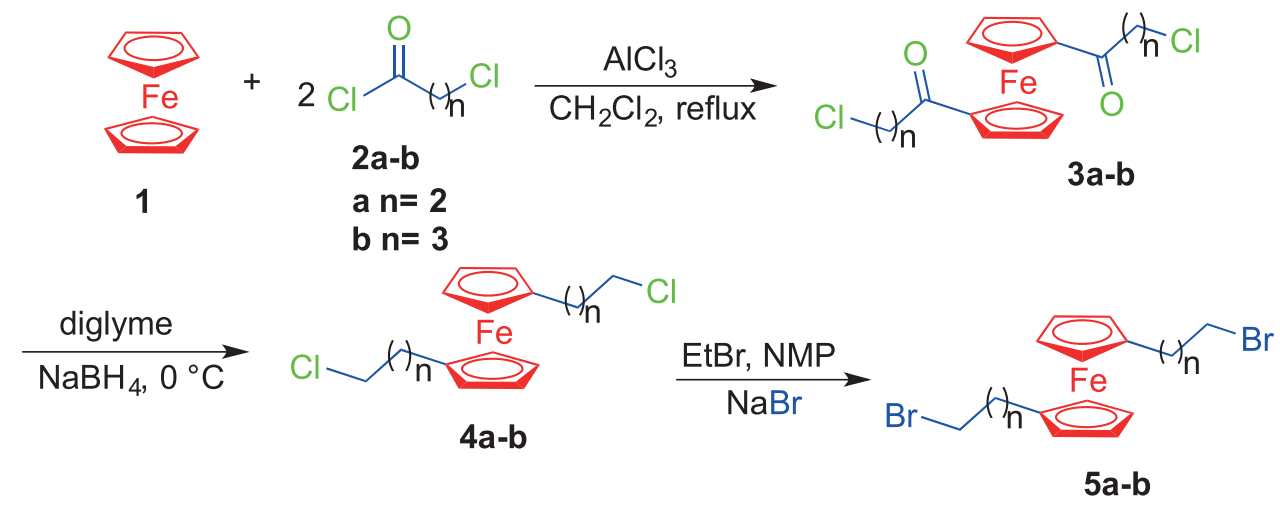

Scheme 1. Synthesis of $\mathbf{5 a}$ and $\mathbf{5 b}$.

Tris(trimethylsilyl)methane was prepared by the reaction of chlorotrimethylsilane, chloroform and $\mathrm{Li}$ in THF. Tris(trimethylsilyl)methane reacted with MeLi under reflux conditions in THF to produce $\left(\mathrm{Me}_{3} \mathrm{Si}\right)_{3} \mathrm{CLi}$ (Scheme 2). ${ }^{50}$ The precursor $\left(\mathrm{HMe}_{2} \mathrm{Si}\right)_{3} \mathrm{CH}$ was made by the reaction of $\mathrm{CHBr}_{3}$ and $\mathrm{Mg}$ with $\mathrm{HMe}_{2} \mathrm{SiCl}$ in $\mathrm{THF},{ }^{45,51}$ which resulted in $\left(\mathrm{HMe}_{2} \mathrm{Si}\right)_{3} \mathrm{CLi}$ after treatment with LDA (lithium diisopropylamide) at room temperature.

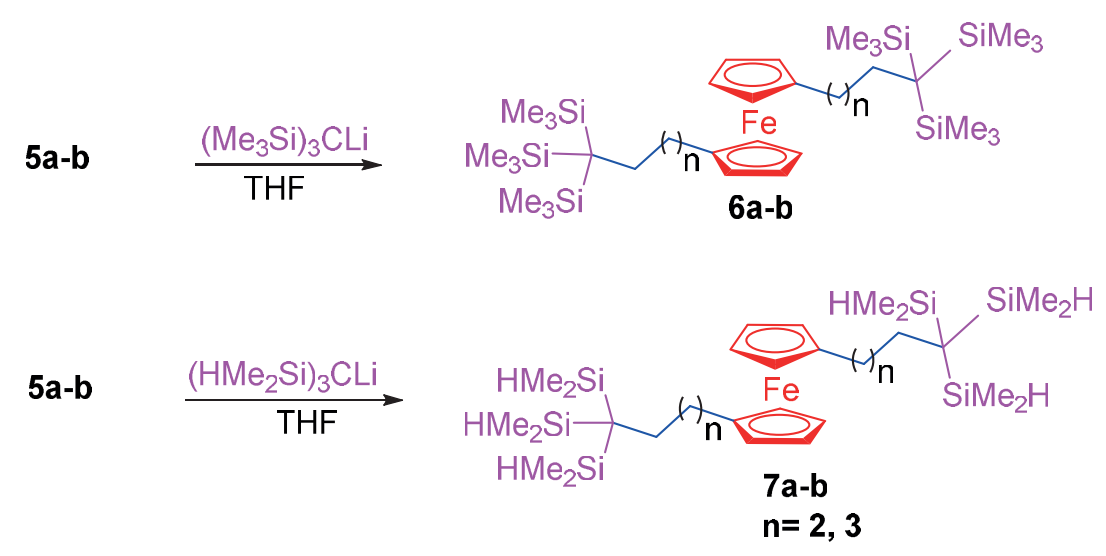

Scheme 2. Synthesis of $6 a-b$ and $7 \mathbf{a}-\mathbf{b}$.

1,1'-Bis(bromoalkyl)ferrocenes $\mathbf{5 a - b}$ were converted into 1,1' bis[tris(trimethylsilyl)methyl]alkylferrocenes (6a-b) and 1,1'-bis [tris(dimethylsilyl)methyl]alkylferrocenes $(\mathbf{7 a - b})$ by treatment with $\left(\mathrm{Me}_{3} \mathrm{Si}\right)_{3} \mathrm{CLi}$ and $\left(\mathrm{HMe}_{2} \mathrm{Si}\right)_{3} \mathrm{CLi}$, respectively, in THF at $0{ }^{\circ} \mathrm{C}$ in good to high yields (Table 1). 
Table 1. Synthesis of compounds $6 \mathbf{a}-\mathbf{b}$ and $7 \mathbf{a}-\mathbf{b}$

\begin{tabular}{cccccc}
\hline Entry & compounds & Reagent & products & Time(h) & ${\text { Yields }(\%)^{\mathrm{a}}}^{\text {(5) }}$ \\
\hline 1 & $\mathbf{5 a}$ & $\left(\mathrm{Me}_{3} \mathrm{Si}\right)_{3} \mathrm{CLi}$ & $\mathbf{6 a}$ & 6 & 90 \\
2 & $\mathbf{5 b}$ & $\left(\mathrm{Me}_{3} \mathrm{Si}\right)_{3} \mathrm{CLi}$ & $\mathbf{6 b}$ & 6 & 92 \\
3 & $\mathbf{5 a}$ & $\left(\mathrm{HMe}_{2} \mathrm{Si}\right)_{3} \mathrm{CLi}$ & $\mathbf{7 a}$ & 8 & 87 \\
4 & $\mathbf{5 b}$ & $\left(\mathrm{HMe}_{2} \mathrm{Si}\right)_{3} \mathrm{CLi}$ & $\mathbf{7 b}$ & 8 & 90 \\
\hline
\end{tabular}

${ }^{\mathrm{a}}$ Isolated yields.

As shown by the results in Table 2, for the synthesis of the target molecule 8a, the conversion was tested in various solvents such as THF and acetone ${ }^{52}$ in the presence of Karstedt catalyst, but was not complete at room temperature or under reflux condition after 12 hours. Methanol was tried as both the solvent and the reagent in this reaction, but at room temperature, no desired product was obtained after 12 hours. However, under reflux conditions the desired polysilyl ether 8a was proiduced after 6 hours in excellent yield (Entry 1, Table 3). Therefore, this reaction was performed with other alcohols as reagent and solvent with heating at $60-80{ }^{\circ} \mathrm{C}$ to give the desired polysilyl ethers in good to excellent yields (Table 3).

Table 2. Optimization of reaction conditions for synthesis of $\mathbf{8 a}$

\begin{tabular}{ccccc}
\hline Entry & Solvent & Condition & Time $/ \mathrm{h}$ & Yield/\% \\
\hline 1 & THF & rt & 12 & - \\
2 & THF & reflux & 12 & - \\
3 & acetone & rt & 12 & - \\
4 & methanol & rt & 12 & - \\
5 & methanol & reflux & 6 & 92 \\
\hline
\end{tabular}

Table 3. Synthesis of 1,1'-bis[tris(alkoxydimethylsilyl)methyl]alkylferrocenes ${ }^{\mathrm{a}}$

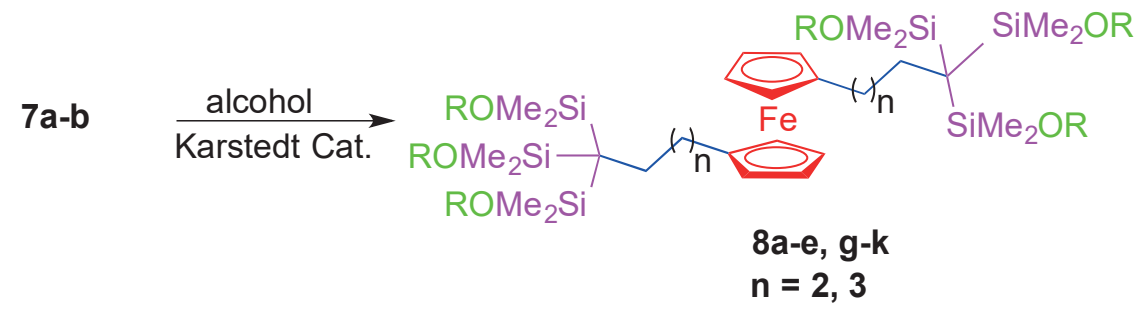

\begin{tabular}{ccccc}
\hline Entry & Compounds & Alcohol & Products & Yield $(\%)^{b}$ \\
\hline 1 & $\mathbf{7 a}$ & $\mathrm{CH}_{3} \mathrm{OH}$ & $\mathbf{8 a}$ & 90 \\
2 & $\mathbf{7 a}$ & $\mathrm{CH}_{3} \mathrm{CH}_{2} \mathrm{OH}$ & $\mathbf{8 b}$ & 85 \\
3 & $\mathbf{7 a}$ & $\mathrm{CH}_{3} \mathrm{CH}_{2} \mathrm{CH}_{2} \mathrm{OH}$ & $\mathbf{8 c}$ & 82 \\
4 & $\mathbf{7 a}$ & $\mathrm{CH}_{3}\left(\mathrm{CH}_{2}\right)_{3} \mathrm{OH}$ & $\mathbf{8 d}$ & 80 \\
\hline
\end{tabular}


Table 3 (continued)

\begin{tabular}{ccccc}
\hline Entry & Compounds & Alcohol & Products & Yield (\%)b \\
\hline 5 & $\mathbf{7 a}$ & $\mathrm{PhCH}_{2} \mathrm{OH}$ & $\mathbf{8 e}$ & 89 \\
6 & $\mathbf{7 a}$ & $\mathrm{PhOH}$ & $\mathbf{8 f}$ & - \\
7 & $\mathbf{7 b}$ & $\mathrm{CH}_{3} \mathrm{OH}$ & $\mathbf{8 g}$ & 92 \\
8 & $\mathbf{7 b}$ & $\mathrm{CH}_{3} \mathrm{CH}_{2} \mathrm{OH}$ & $\mathbf{8 h}$ & 89 \\
9 & $\mathbf{7 b}$ & $\mathrm{CH}_{3} \mathrm{CH}_{2} \mathrm{CH}_{2} \mathrm{OH}$ & $\mathbf{8 i}$ & 86 \\
10 & $\mathbf{7 b}$ & $\mathrm{CH}_{3}\left(\mathrm{CH}_{2}\right)_{3} \mathrm{OH}$ & $\mathbf{8 j}$ & 83 \\
11 & $\mathbf{7 b}$ & $\mathrm{PhCH}_{2} \mathrm{OH}$ & $\mathbf{8 k}$ & 92 \\
\hline
\end{tabular}

${ }^{a}$ reaction conditions: $7 \mathbf{a}-\mathbf{b}(0.20 \mathrm{~g})$ and $\mathrm{ROH}(20 \mathrm{~mL})$, Karstedt catalyst $([\mathrm{Pt}] /[\mathrm{Si}-\mathrm{H}]=7.2 \times 10-3)$ under dry argon at $60-80{ }^{\circ} \mathrm{C} .{ }^{\mathrm{b}}$ Isolated yields

Primary aliphatic alcohols with long alkyl chains were less effective than those with short chains (Table 3). Alcohols with an aromatic ring such as benzyl alcohol gave the corresponding silyl ethers in high yields (Entry 5, Table 3, 92\%), but phenols failed to give the desired phenolic compound (Entry 6 Table 3). We also used secondary alcohols in this reaction but the reaction did not go to completion, and our attempt at the synthesis of branched polysilyl ether with secondary alcohols failed and one or two alcohols react with Si-H bound in each side. Tertiary alcohols such as tert-butanol did not react with the silane. Also, attempted use of kojic acid as a biological alcohol, was unsuccessful in this reaction.

The ${ }^{1} \mathrm{HNMR}$ spectrum of compound $\mathbf{8 b}$ (Figure 1) shows the signal for the ferrocenyl protons at $3.95 \mathrm{ppm}$, the singlet ascribed to $\mathrm{SiMe}_{2}$ at $0.18 \mathrm{ppm}, \mathrm{CH}_{2} \mathrm{O}$ protons at 3.57-3.62, and $\mathrm{CH}_{3}$ protons at 1.12-1.15 ppm. 


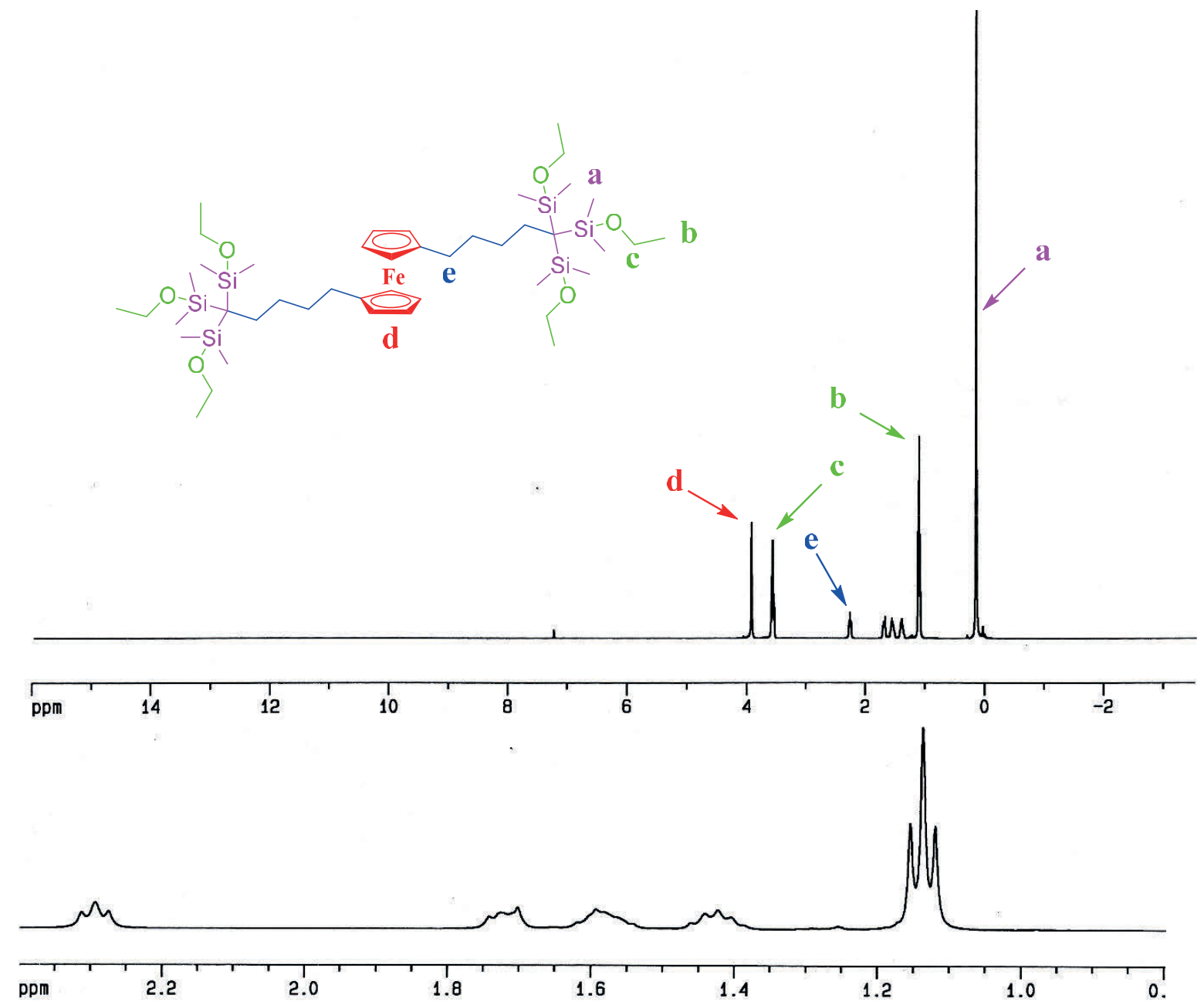

Figure 1. ${ }^{1} \mathrm{HNMR}$ spectra of $1,1^{\prime}$-bis[4-(tris(ethoxydimethylsilyl)methyl)butyl]ferrocene.

\section{Conclusions}

In summary, we report the synthesis of 1,1'-bis[tris(trimethylsilyl)methyl]alkylferrocenes and 1,1'-bis[tris(dimethylsilyl)methyl]alkylferrocenes from treatment of $\left(\mathrm{Me}_{3} \mathrm{Si}\right)_{3} \mathrm{CLi}$ and $\left(\mathrm{HMe}_{2} \mathrm{Si}\right)_{3} \mathrm{CLi}$ with $1,1^{\prime}$-bis(bromoalkyl)ferrocene derivatives (5a-b).

1,1'-[Tris(alkoxydimethylsilyl)methyl]alkylferrocenes were prepared by dehydrocoupling reactions of hydrosilane derivatives with various aliphatic and benzylic alcohols in the presence of the Karstedt catalyst (platinum(0)-1,3-divinyl-1,1,3,3-tetramethyldisiloxane in xylene). These products are potentially capable of various applications such as dendrimer cores, nano materials, macromolecules and intermediates in organic synthesis. 


\section{Experimental Section}

General. Chemicals were either prepared in our laboratory or purchased from Merck, Fluka and Aldrich. Commercial products were used without purification.

The ${ }^{1} \mathrm{H}$ and ${ }^{13} \mathrm{C}$ NMR spectra were recorded with a Bruker FT-400 MHz spectrometer at room temperature and with $\mathrm{CDCl}_{3}$ as solvent. The FTIR spectra were recorded on a Bruker-Tensor 270 spectrometer. The mass spectra were obtained with a GC-Mass Agilent quadrupole mode 5973N instrument, operating at $70 \mathrm{eV}$. Elemental analyses were carried out with an Elementar vario EL III instrument.

Preparation of 1,1'-bis(chloroalkyl)ferrocenes (4a-4b). A solution of 4-chlorobutanoyl chloride (15.28 g, $108 \mathrm{mmol}$ ) or 3-chloropropionyl chloride (13.60 g, $108 \mathrm{mmol})$ in $50 \mathrm{~mL}$ dry $\mathrm{CH}_{2} \mathrm{Cl}_{2}$ was added drop wise to a suspension of anhydrous $\mathrm{AlCl}_{3}(15.89 \mathrm{~g}, 119 \mathrm{mmol})$ in $100 \mathrm{~mL}$ dry $\mathrm{CH}_{2} \mathrm{Cl}_{2}$ at $\mathrm{rt}$. The mixture was stirred at $\mathrm{rt}$ for $1 \mathrm{~h}$ under argon. The obtained homogeneous yellow solution was added dropwise to a solution of ferrocene $(54 \mathrm{mmol})$ in dry $\mathrm{CH}_{2} \mathrm{Cl}_{2}(130 \mathrm{~mL})$ at $0{ }^{\circ} \mathrm{C}$. The solution turned to dark purple, and was refluxed for $8 \mathrm{~h}$, and then a solution of $\mathrm{NaBH}_{4}(4.09 \mathrm{~g}, 108$ $\mathrm{mmol})$ in diglyme $(50 \mathrm{~mL})$ was added to the mixture while maintaining the solution temperature at less than or equal to $0{ }^{\circ} \mathrm{C}$. A dark orange solution was formed and stirred at $0{ }^{\circ} \mathrm{C}$ for $1 \mathrm{~h}$. The mixture was hydrolyzed by the addition of $\mathrm{H}_{2} \mathrm{O}(120 \mathrm{~mL})$ while keeping its temperature at 0 to $5{ }^{\circ} \mathrm{C}$. The mixture was allowed to separate by settling and the organic phase, was withdrawn. The aqueous phase was extracted by $\mathrm{CH}_{2} \mathrm{Cl}_{2},(50 \mathrm{~mL})$ which was repeated 3 times and then all the organic phases were combined and washed with brine $(100 \mathrm{~mL})$. The $\mathrm{CH}_{2} \mathrm{Cl}_{2}$ was distilled off under atmospheric pressure and the diglyme was distilled off under reduced pressure. The residue was purified by column chromatography on silica gel with $n$-hexane as eluant.

1,1'-Bis(3-chloropropyl)ferrocene (4a). Brown oil (90\%); FTIR $\left(v_{\max }, \mathrm{cm}^{-1}\right)$ : $3084(\mathrm{Cp}-\mathrm{H})$, 2932, $2853(\mathrm{C}-\mathrm{H}), 1650,1441(\mathrm{C}=\mathrm{C}), 1039,495(\mathrm{Cp}), 815\left(\mathrm{CH}_{2}-\mathrm{Cl}\right) ;{ }^{1} \mathrm{HNMR}\left(400 \mathrm{MHz}, \mathrm{CDCl}_{3}\right)$ $\delta=1.91-1.98\left(\mathrm{~m}, 4 \mathrm{H}, J 6.5 \mathrm{~Hz}, \mathrm{Cp}-\mathrm{CH}_{2}-\mathrm{CH}_{2}\right), 2.47-2.51\left(\mathrm{t}, 4 \mathrm{H}, J 7.6 \mathrm{~Hz}, \mathrm{Cp}-\mathrm{CH}_{2}\right), 3.52-3.55$ (t, $\left.4 \mathrm{H}, J 6.5 \mathrm{~Hz}, \mathrm{CH}_{2}-\mathrm{Cl}\right), 4.01-4.04(\mathrm{~d}, 8 \mathrm{H}, J 8.1 \mathrm{~Hz}, \mathrm{Cp}) ;{ }^{13} \mathrm{CNMR}\left(100 \mathrm{MHz}, \mathrm{CDCl}_{3}\right) \delta=26.6$, 32.7, (- $\left.\mathrm{CH}_{2}-\right), 32.9\left(\mathrm{CH}_{2}-\mathrm{Cl}\right), 67.7,67.1(\mathrm{Cp}), 86.1\left(\mathrm{C}_{1} \mathrm{Cp}\right) ; \mathrm{m} / z(\mathrm{EI}) 338[\mathrm{M}]^{+}, 340[\mathrm{M}+2]^{+}$. Calc. for $\mathrm{C}_{16} \mathrm{H}_{20} \mathrm{Cl}_{2} \mathrm{Fe}$ : C, 56.68; H 5.95. Found: C, 56.53; H, 5.91\%.

1,1'-Bis(4-chlorobutyl)ferrocene (4b). Brown oil (92\% yield). FTIR ( $\left.\mathrm{KBr}, \mathrm{cm}^{-1}\right): v=3085$ (Cp$\mathrm{H}), 2936,2857$ (C-H), 1640, 1445 (C=C), 1105, 492 (Cp), 819 (C-Cl); ${ }^{1} \mathrm{HNMR}\left(400 \mathrm{MHz}, \mathrm{CDCl}_{3}\right)$ $\delta=1.62-1.66\left(\mathrm{~m}, 4 \mathrm{H}, J 8 \mathrm{~Hz}, \mathrm{Cp}-\mathrm{CH}_{2}-\mathrm{CH}_{2}\right), 1.76-1.82\left(\mathrm{~m}, 4 \mathrm{H}, J 6.5 \mathrm{~Hz}, \mathrm{CH}_{2}-\mathrm{CH}_{2}-\mathrm{Cl}\right), 2.33-2.37$ (t, $\left.J 7.6 \mathrm{~Hz}, 4 \mathrm{H}, \mathrm{Cp}-\mathrm{CH}_{2}\right), 3.53-3.56$ (t, $\left.4 \mathrm{H}, \mathrm{J} 6.6 \mathrm{~Hz} \mathrm{CH}_{2}-\mathrm{Cl}\right), 3.98-4.01$ (d, 8H, Cp); ${ }^{13} \mathrm{CNMR}$ $\left(100 \mathrm{MHz}, \mathrm{CDCl}_{3}\right) \delta=27.3,27.6,31.28\left(-\mathrm{CH}_{2}-\right), 43.9\left(\mathrm{CH}_{2}-\mathrm{Cl}\right), 66.8,67.5(\mathrm{Cp}), 87.4\left(\mathrm{C}_{1} \mathrm{Cp}\right) ; \mathrm{m} / \mathrm{z}$ (EI) $366[\mathrm{M}]^{+}, 368[\mathrm{M}+2]^{+}$. Calc. for $\mathrm{C}_{18} \mathrm{H}_{24} \mathrm{Cl}_{2} \mathrm{Fe}$ : C, 58.89; H 6.59. Found: C, 58.75; H, 6.53\%. Preparation of 1,1'-bis(bromoalkyl)ferrocene (5a-5b). A mixture of $0.01 \mathrm{~mol}$ of 1,1'bis(bromoalkyl)ferrocene derivatives, $\operatorname{EtBr}(20 \mathrm{~mL})$, and $\mathrm{NaBr}(0.24 \mathrm{~g}, 0.004 \mathrm{~mol})$ in NMP (30 $\mathrm{mL}$ ) was heated at $65{ }^{\circ} \mathrm{C}$ for 5 days. The mixture was allowed to cool and then was poured into a 
mixture of ice-water, and brine, in a separatory funnel. The lower layer was removed and the organic layer washed with brine and distilled water. The solvent was removed and the product was purified by column chromatography on silica. A brown band was eluted with a hexane/EtOAc $(6: 2)$ mixture to give the product.

1,1'-Bis(3-bromopropyl)ferrocene (5a). From 1,1'-bis(3-chloropropyl)ferrocene (4 g), a brown oil was obtained (4.63 g, 92\%). FTIR (KBr, cm $\left.{ }^{-1}\right): v=3085(\mathrm{Cp}-\mathrm{H}), 2931,2848(\mathrm{C}-\mathrm{H}), 1638$, $1437(\mathrm{C}=\mathrm{C}), 1029,497,(\mathrm{Cp}), 820(\mathrm{C}-\mathrm{Br}) ;{ }^{1} \mathrm{HNMR}\left(400 \mathrm{MHz}, \mathrm{CDCl}_{3}\right) \delta=1.99-2.06(\mathrm{~m}, 4 \mathrm{H}, J 6.6$ $\mathrm{Hz}, \mathrm{CpCH}_{2}-\mathrm{CH}_{2}$ ), 2.47-2.51 (t, 4H, J 7.5 Hz, Cp-CH2), 3.39-3.42 (t, 4H, J 6.5 Hz, $\mathrm{CH}_{2}-\mathrm{Br}$ ), 4.014.04 (d, 8H, Cp); ${ }^{13} \mathrm{CNMR}\left(100 \mathrm{MHz}, \mathrm{CDCl}_{3}\right) \delta=26.6,32.7,32.9\left(-\mathrm{CH}_{2^{-}}\right), 67.0,67.7(\mathrm{Cp}), 86.1$ $\left(\mathrm{C}_{1} \mathrm{Cp}\right) ; \mathrm{m} / z(\mathrm{EI}) 426[\mathrm{M}]^{+}, 428[\mathrm{M}+2]^{+}$. Calc. for $\mathrm{C}_{16} \mathrm{H}_{20} \mathrm{Br}_{2} \mathrm{Fe}: \mathrm{C}, 44.90 ; \mathrm{H}$ 4.71. Found: $\mathrm{C}, 44.79$; $\mathrm{H}, 4.65 \%$.

1,1'-Bis(4-bromobutyl)ferrocene (5b). From 1,1'-bis(4-chlorobutyl)ferrocene (4.0 g), a brown oil $(4.51 \mathrm{~g}, 91 \%)$ was obtained. FTIR $\left(\mathrm{KBr}, \mathrm{cm}^{-1}\right): v=3085(\mathrm{Cp}-\mathrm{H}), 2933,2854(\mathrm{C}-\mathrm{H}), 1643$, $1439(\mathrm{C}=\mathrm{C}), 1105,1028,491(\mathrm{Cp}), 818(\mathrm{C}-\mathrm{Br}) ;{ }^{1} \mathrm{HNMR}\left(400 \mathrm{MHz}, \mathrm{CDCl}_{3}\right) \delta=1.60-1.68(\mathrm{~m}, 4 \mathrm{H}$, $\left.J 7.2 \mathrm{~Hz}, \mathrm{Cp}-\mathrm{CH}_{2}-\mathrm{CH}_{2}\right), 1.84-1.91\left(\mathrm{~m}, 4 \mathrm{H}, J 6.8 \mathrm{~Hz}, \mathrm{CH}_{2}-\mathrm{CH}_{2}-\mathrm{Br}\right), 2.32-2.36$ (t, $4 \mathrm{H}, J 7.7 \mathrm{~Hz}, \mathrm{Cp}-$ $\mathrm{CH}_{2}$ ), 3.39-3.43 (t, $\left.4 \mathrm{H}, J 6.7 \mathrm{~Hz}, \mathrm{CH}_{2}-\mathrm{Br}\right), 3.97-3.99$ (d, 8H, Cp); ${ }^{13} \mathrm{CNMR}\left(100 \mathrm{MHz}, \mathrm{CDCl}_{3}\right) \delta$ $=27.5,28.6,31.4,32.8\left(-\mathrm{CH}_{2^{-}}\right), 66.8,67.6(\mathrm{Cp}), 87.4\left(\mathrm{C}_{1} \mathrm{Cp}\right) ; \mathrm{m} / z(\mathrm{EI}) 454[\mathrm{M}]^{+}, 456[\mathrm{M}+2]^{+}$, $199\left[\mathrm{FcCH}_{2}\right]^{+}, 121[\mathrm{CpFe}]^{+}$. Anal. Calc. for $\mathrm{C}_{18} \mathrm{H}_{24} \mathrm{Br}_{2} \mathrm{Fe}$ : C, 47.41; H, 5.30. Found: C, 47.35; H, $5.24 \%$.

Preparation of tris(trimethylsilyl)methyllithium, $\left(\mathrm{Me}_{3} \mathrm{Si}\right)_{3} \mathrm{CLi}$. The reagent was prepared as described by Gröbel and co-workers. ${ }^{47}$

Preparation of tris(dimethylsilyl)methyllithium, $\left(\mathrm{HSiMe}_{2}\right)_{3} \mathbf{C L i}$. A $50 \mathrm{~mL}$ round-bottom flask equipped with a stirrer, septum, and a gas-inlet needle was charged with $i$ - $\operatorname{Pr}_{2} \mathrm{NH}(1.06 \mathrm{~g}, 10.6$ $\mathrm{mmol})$ and THF $(20 \mathrm{~mL})$. The flask was placed in a water ice bath and then $n$-BuLi $(7.6 \mathrm{~mL}, 1.5$ $\mathrm{M}$ solution in hexane) was added dropwise to the stirring mixture to form a clear yellow solution. The solution was stirred for an additional $30 \mathrm{~min}$. The lithium diisopropylamide (LDA) solution was transferred into a dropping funnel then added dropwise to a $50 \mathrm{~mL}$ round-bottom flask containing tris(dimethylsilyl)methane, $\left(\mathrm{HSiMe}_{2}\right)_{3} \mathrm{CH},(2.0 \mathrm{~g}, 10.6 \mathrm{mmol})$, in THF $(20 \mathrm{~mL})$ under argon at rt. Finally the orange-red solution was stirred at ambient temperature for $10 \mathrm{~h}$.

Preparation of compounds (6a-b) and (7a-b). To a stirred solution of $\left(\mathrm{Me}_{3} \mathrm{Si}\right)_{3} \mathrm{CLi}$ or $\left(\mathrm{HSiMe}_{3}\right)_{3} \mathrm{CLi}(5.3 \mathrm{mmol})$ in THF at $0{ }^{\circ} \mathrm{C}$ was added 1,1'-bis(3-bromopropyl)ferrocene 5a or 1,1'bis(4-bromobutyl)ferrocene $\mathbf{5 b}(5.0 \mathrm{mmol})$ in THF $(10 \mathrm{~mL})$, and the mixture was stirred for 6-8 $\mathrm{h}$ at rt. It was then poured into aq $\mathrm{NH}_{4} \mathrm{Cl}(50 \mathrm{~mL})$ and extracted with $\mathrm{CH}_{2} \mathrm{Cl}_{2}(2 \times 50 \mathrm{~mL})$. The organic phase was washed with $\mathrm{H}_{2} \mathrm{O}(100 \mathrm{~mL})$ and dried $\left(\mathrm{Na}_{2} \mathrm{SO}_{4}\right)$, and the solvent was removed to leave a viscous oil.

1,1'-Bis[3-(tris(trimethylsilyl)methyl)propyl]ferrocene (6a). From 1,1'-bis(3bromopropyl)ferrocene (3.0 g), a yellowish oil (4.62 g, 90\%) of was obtained. FTIR $\left(\mathrm{KBr}, \mathrm{cm}^{-1}\right)$ : 
$v=3085(\mathrm{Cp}-\mathrm{H}), 2952,2844(\mathrm{C}-\mathrm{H})$, 1645, $1454(\mathrm{C}=\mathrm{C})$, 1254, $838(\mathrm{Si}-\mathrm{C}), 1025,956,496(\mathrm{Fc})$; ${ }^{1} \mathrm{HNMR}\left(400 \mathrm{MHz}, \mathrm{CDCl}_{3}\right) \delta=0.1\left(\mathrm{~s}, 54 \mathrm{H}, \mathrm{SiMe}_{3}\right), 1.65-1.69\left(\mathrm{~m}, 8 \mathrm{H}, J 7 \mathrm{~Hz},-\mathrm{CH}_{2}-\right), 2.24-2.27$ (t, 4H, J 7.2 Hz, Cp-CH2), 3.94-3.97 (d, 8H, Cp); ${ }^{13} \mathrm{CNMR}\left(100 \mathrm{MHz}, \mathrm{CDCl}_{3}\right) \delta=2.0\left(\mathrm{SiMe}_{3}\right)$, $4.8\left(\mathrm{C}\left(\mathrm{SiMe}_{3}\right)_{3}\right), 29.7,29.9,30.3\left(-\mathrm{CH}_{2}-\right), 66.6,67.4(\mathrm{Cp}), 87.6\left(\mathrm{C}_{1} \mathrm{Cp}\right) ; \mathrm{m} / z(\mathrm{EI}) 730[\mathrm{M}]^{+}$. Anal. Calc. for $\mathrm{C}_{36} \mathrm{H}_{74} \mathrm{FeSi}_{6}$ : C, 59.12; H 10.20. Found: C, 59.02; H, 10.11\%.

\section{1,1'-Bis[4-(tris(trimethylsilyl)methyl)butyl]ferrocene $\quad(6 \mathbf{b}) . \quad$ From 1,1'-bis(4-} bromobutyl)ferrocene $(3.0 \mathrm{~g})$, a yellowish oil $(4.6 \mathrm{~g}, 92 \%)$ was obtained. FTIR $\left(\mathrm{KBr}, \mathrm{cm}^{-1}\right): v=$ 3086 (Cp-H), 2952, 2848 (C-H), 1626, 1441 (C=C), 1255, 842 (Si-C), 1039, 495 (Cp), 484 (Fc); ${ }^{1} \mathrm{HNMR}\left(400 \mathrm{MHz}, \mathrm{CDCl}_{3}\right) \delta=0.1\left(\mathrm{~s}, 54 \mathrm{H}, \mathrm{SiMe}_{3}\right), 1.42-1.54\left(\mathrm{~m}, 8 \mathrm{H}, J 7.2 \mathrm{~Hz},-\mathrm{CH}_{2}-\right), 1.54-$ 1.61 (t, 4H, $\left.-\mathrm{CH}_{2} \mathrm{C}\left(\mathrm{SiMe}_{3}\right)_{3}\right), 2.29-2.32$, (t, $\left.4 \mathrm{H}, J 7 \mathrm{~Hz}, \mathrm{Cp}-\mathrm{CH}_{2}\right), 3.95-3.96$ (d, 8H, Cp); ${ }^{13} \mathrm{CNMR}$ $\left(100 \mathrm{MHz}, \mathrm{CDCl}_{3}\right) \delta=1.8\left(\mathrm{SiMe}_{3}\right), 5.1\left(\mathrm{C}\left(\mathrm{SiMe}_{3}\right)_{3}\right), 28.4,29.1,30.0,31.9\left(-\mathrm{CH}_{2}-\right), 66.7,67.5$ $(\mathrm{Cp}), 88.0\left(\mathrm{C}_{1} \mathrm{Cp}\right) ; m / z(\mathrm{EI}) 758[\mathrm{M}]^{+}$. Anal. Calc. for $\mathrm{C}_{38} \mathrm{H}_{78} \mathrm{FeSi}_{6}: \mathrm{C}, 60.10 ; \mathrm{H}$ 10.35. Found: C, $60.01 ; \mathrm{H}, 10.29 \%$.

1,1'-Bis[3-(tris(dimethylsilyl)methyl)propyl)ferrocene (7a). From 1,1'-bis(3bromopropyl)ferrocene (3.0 g), a yellowish oil (3.95 g, 87\%) was obtained. FTIR $\left(\mathrm{KBr}, \mathrm{cm}^{-1}\right): v$ $=3085(\mathrm{Cp}-\mathrm{H})$, 2955, $2903(\mathrm{C}-\mathrm{H}), 2106(\mathrm{Si}-\mathrm{H}), 1589(\mathrm{Cp})$, 1253, 849 (Si-C), 1054, 438; ${ }^{1} \mathrm{HNMR}$ $\left(400 \mathrm{MHz}, \mathrm{CDCl}_{3},\right) \delta=0.16-0.17\left(\mathrm{~d}, 36 \mathrm{H}, J 3.7 \mathrm{~Hz}, \mathrm{SiMe}_{2}\right), 1.65-1.75\left(\mathrm{~m}, 8 \mathrm{H},-\mathrm{CH}_{2^{-}}\right), 2.25-2.29$ (t, 4H, J 7.5 Hz, Cp-CH2), 3.94-3.98 (dd, $J$ 1.5, 8H, Cp), 4.00-4.05 (m, J 3.7 Hz, 6H, Si-H); ${ }^{13} \mathrm{CNMR}\left(100 \mathrm{MHz}, \mathrm{CDCl}_{3}\right) \delta=-4.1\left(\mathrm{SiMe}_{2}\right), 0.36\left(\mathrm{C}\left(\mathrm{SiMe}_{2}\right)_{3}\right), 29.0,29.4,29.5\left(-\mathrm{CH}_{2}-\right), 66.6$, $67.5(\mathrm{Cp}), 87.7\left(\mathrm{C}_{1} \mathrm{Cp}\right) ; \mathrm{m} / z(\mathrm{EI}): 646[\mathrm{M}]^{+}$. Anal. Calc. for $\mathrm{C}_{30} \mathrm{H}_{62} \mathrm{FeSi}_{6}$ : C, 55.68; H 9.66. Found: C, $55.55 ; \mathrm{H}, 9.59 \%$.

1,1'-Bis[4-(tris(dimethylsilyl)methyl)butyl)ferrocene (7b). From 1,1'-[4-bromobutyl]ferrocene (3.0 g), a yellowish oil (4 g, 90\%) was obtained. FTIR $\left(\mathrm{KBr}, \mathrm{cm}^{-1}\right): v=3087(\mathrm{Cp}), 2933,2851(\mathrm{C}-$ H), 2107 (Si-H), 1642, 1425 (Cp), 1253, 839 (Si-C), 1028, 889, 493 (Cp); ${ }^{1} \mathrm{HNMR}$ (400 MHz, $\left.\mathrm{CDCl}_{3}\right) \delta=0.15-0.16\left(\mathrm{~d}, J 3.1 \mathrm{~Hz}, 36 \mathrm{H}, \mathrm{SiMe}_{2}\right), 1.43-1.48\left(\mathrm{~m}, 8 \mathrm{H},-\mathrm{CH}_{2}-\right), 1.54-1.63(\mathrm{t}, 4 \mathrm{H}$, $\left.\mathrm{CH}_{2} \mathrm{C}\left(\mathrm{SiMe}_{2} \mathrm{H}\right)_{3}\right), 2.29-2.33$, (t, 4H, J 7.6 Hz, Cp-CH $), 3.95-3.96$, (d, 8H Cp), 4.00-4.03 (m, J 3.5 $\mathrm{Hz}, 6 \mathrm{H}, \mathrm{Si}-H) ;{ }^{13} \mathrm{CNMR}\left(100 \mathrm{MHz}, \mathrm{CDCl}_{3}\right) \delta=-4.1\left(\mathrm{SiMe}_{2}\right), 0.40\left(\mathrm{C}\left(\mathrm{SiMe}_{2}\right)_{3}\right), 28.2,28.3,29.0$, $31.4\left(-\mathrm{CH}_{2}-\right), 68.2,67.5(\mathrm{Cp}), 87.4\left(\mathrm{C}_{1} \mathrm{Cp}\right) ; \mathrm{m} / z(\mathrm{EI}): 674[\mathrm{M}]^{+}$. Anal. Calc. for $\mathrm{C}_{32} \mathrm{H}_{66} \mathrm{FeSi}_{6}: \mathrm{C}_{\text {, }}$ 56.92; H 9.58. Found: C, 56.83; H, 9.43\%.

General procedure for the synthesis of $1,1^{\prime}$ bis[(tris(methoxydimethylsilyl)methyl)alkyl]ferrocene. A $50 \mathrm{~mL}$ round-bottom two-neck flask with magnetic stirrer was charged with $7 \mathbf{a}$ or $7 \mathbf{b}(0.20 \mathrm{~g}, 0.29 \mathrm{mmol} 7 \mathbf{a}$ and $0.3 \mathrm{mmol} 7 \mathbf{b})$ and ROH $(20 \mathrm{~mL})$ under dry argon. Karstedt catalyst $([\mathrm{Pt}] /[\mathrm{Si}-\mathrm{H}]=7.2 \times 10-3)$ was added and the reaction progress was monitored. Several samples were taken over reaction time and were analyzed by FTIR spectroscopy. The mixture was stirred at $60-80{ }^{\circ} \mathrm{C}$ until complete disappearance of the $\mathrm{Si}-\mathrm{H}$ peak in the FTIR spectra. After completion of the reaction, the mixture was allowed to cool to rt., then the alcohol was evaporated under reduced pressure and the residue was purified by flash column chromatography (silica gel, 10:1 $n$-hexane:EtOAc) to give a highly viscous oily product. 1,1'-Bis[3-(tris(methoxydimethylsilyl)methyl)propyl]ferrocene (8a). Yellowish oil, FTIR 
$\left(\mathrm{KBr}, \mathrm{cm}^{-1}\right): v=3096(\mathrm{Cp}-\mathrm{H}), 2925(\mathrm{C}-\mathrm{H}), 1638,1463(\mathrm{C}=\mathrm{C})$, 1252, 833(C-Si), 1093, $488(\mathrm{Cp})$, 1002 (Si-O); ${ }^{1} \mathrm{HNMR}\left(400 \mathrm{MHz}, \mathrm{CDCl}_{3}\right) \delta=0.21$ (s, $\left.18 \mathrm{H}, \mathrm{SiMe}_{2}\right), 1.76-1.79\left(\mathrm{~m}, 8 \mathrm{H},-\mathrm{CH}_{2^{-}}\right)$, 2.22-2.24 (t, 4H, J 7.2 Hz, Cp- $\mathrm{CH}_{2}$ ), 3.37 (s, 18H, OCH $), 3.95-3.96,(\mathrm{~d}, 8 \mathrm{H}, \mathrm{Cp}) ;{ }^{13} \mathrm{CNMR}(100$ $\left.\mathrm{MHz}, \mathrm{CDCl}_{3}\right) \delta=-0.92\left(\mathrm{SiMe}_{2}\right), 16.0,27.5,29.7,30.4\left(-\mathrm{CH}_{2^{-}}\right), 48.9\left(\mathrm{OCH}_{3}\right), 65.5,67.4(\mathrm{Cp})$, $88.1\left(\mathrm{C}_{1} \mathrm{Cp}\right)$; Anal. Calc. for $\mathrm{C}_{36} \mathrm{H}_{74} \mathrm{FeO}_{6} \mathrm{Si}_{6}$ : C, 52.26; H 9.02. Found: C, 52.13; H, 8.89\%.

1,1'-Bis[3-(tris(ethoxydimethylsilyl)methyl)propyl]ferrocene (8b). Yellowish oil, FTIR (KBr, $\left.\mathrm{cm}^{-1}\right): v=3092(\mathrm{Cp}-\mathrm{H}), 2930,2859(\mathrm{C}-\mathrm{H}), 1633,1458(\mathrm{C}=\mathrm{C}), 1252,853$ (Si-C), 1096, 1030, 485 (Cp), 954 (Si-O); ${ }^{1} \mathrm{HNMR}\left(400 \mathrm{MHz}, \mathrm{CDCl}_{3}\right) \delta=0.2$ (s, 36H, SiMe $)$ ), 1.12-1.15 (t, 18H, J=6.9, $\left.\mathrm{CH}_{3}\right), 1.76-1.86\left(\mathrm{~m}, 8 \mathrm{H},-\mathrm{CH}_{2}-\right), 2.22-2.26\left(\mathrm{t}, 4 \mathrm{H}, J 7.9 \mathrm{~Hz}, \mathrm{Cp}-\mathrm{CH}_{2}\right), 3.58-3.63$ (q, $12 \mathrm{H}, J 6.9 \mathrm{~Hz}$, $\left.\mathrm{OCH}_{2}\right), 3.95-3.96(\mathrm{~d}, 8 \mathrm{H}, \mathrm{Cp}), 4.1 ;{ }^{13} \mathrm{CNMR}\left(100 \mathrm{MHz}, \mathrm{CDCl}_{3}\right) \delta=-0.31\left(\mathrm{SiMe}_{2}\right), 15.4,17.6(-$ $\left.\mathrm{CH}_{3}\right), 27.5,29.7,29.8\left(-\mathrm{CH}_{2}-\right), 63.3\left(\mathrm{OCH}_{2}\right), 66.8,67.4(\mathrm{Cp}), 88.3\left(\mathrm{C}_{1} \mathrm{Cp}\right)$; Anal. Calc. for $\mathrm{C}_{42} \mathrm{H}_{86} \mathrm{FeO}_{6} \mathrm{Si}_{6}$ : C, 55.34; $\mathrm{H}$ 9.51. Found: C, 55.42; H, 9.60\%.

1,1'-Bis[3-(tris(propoxydimethylsilyl)methyl)propyl]ferrocene (8c). Yellowish oil, FTIR $\left(\mathrm{KBr}, \mathrm{cm}^{-1}\right): v=3095(\mathrm{Cp}-\mathrm{H}), 2928,2862(\mathrm{C}-\mathrm{H}), 1632,1465(\mathrm{C}=\mathrm{C}), 1252,837(\mathrm{Si}-\mathrm{C}), 1094,487$ (Cp), 1007 (Si-O); ${ }^{1} \mathrm{HNMR}\left(400 \mathrm{MHz}, \mathrm{CDCl}_{3}\right) \delta=0.21$ (s, 36H, $\mathrm{SiMe}_{2}$ ), 0.88-0.92 (t, 18H, J 7.3 $\left.\mathrm{Hz},-\mathrm{CH}_{3}\right), 1.50-1.57\left(\mathrm{~m}, 12 \mathrm{H},-\mathrm{CH}_{2}-\right), 1.74-1.85(\mathrm{~m}, 8 \mathrm{H}), 2.24-2.28$, (t, $\left.4 \mathrm{H}, J 7.5 \mathrm{~Hz}, \mathrm{Cp}-\mathrm{CH}_{2}\right)$, 3.48-3.51, (t, $\left.12 \mathrm{H}, J 6.5 \mathrm{~Hz}, \mathrm{OCH}_{2}\right), 3.95-3.96(\mathrm{~d}, 8 \mathrm{H}, \mathrm{Cp}) ;{ }^{13} \mathrm{CNMR}\left(100 \mathrm{MHz}, \mathrm{CDCl}_{3}\right) \delta=-0.32$ $\left(\mathrm{SiMe}_{2}\right), 9.6\left(-\mathrm{CH}_{3}\right), 16.0,24.8,27.9,29.8,30.7\left(-\mathrm{CH}_{2}\right), 63.4\left(\mathrm{OCH}_{2}\right), 66.5,67.3(\mathrm{Cp}), 88.3\left(\mathrm{C}_{1}\right.$ $\mathrm{Cp}$ ); Anal. Calc. for $\mathrm{C}_{48} \mathrm{H}_{98} \mathrm{FeO}_{6} \mathrm{Si}_{6}$ : C, 57.90; $\mathrm{H}$ 9.92. Found: C, 57.78; H, 9.86\%.

1,1'-Bis[3-(tris(butoxydimethylsilyl)methyl)propyl]ferrocene (8d). Yellowish oil, FTIR (KBr, $\left.\mathrm{cm}^{-1}\right)$ : $v 3092(\mathrm{Cp}-\mathrm{H}), 2934,2859(\mathrm{C}-\mathrm{H}), 1675,1452(\mathrm{C}=\mathrm{C})$, 1254, $835(\mathrm{Si}-\mathrm{C}), 1109,1009,488$ (Cp), 948 ( $\mathrm{Si}-\mathrm{O}) ;{ }^{1} \mathrm{HNMR}\left(400 \mathrm{MHz}, \mathrm{CDCl}_{3}\right) \delta=0.21$ (s, 36H, SiMe $), 0.88-0.92$ (t, 18H, J 7.2 $\left.\mathrm{Hz},-\mathrm{CH}_{3}\right), 1.32-1.41\left(\mathrm{~m}, 12 \mathrm{H},-\mathrm{CH}_{2}-\right), 1.45-1.50\left(\mathrm{~m}, 12 \mathrm{H},-\mathrm{CH}_{2}-\right), 1.78-1.84\left(\mathrm{~m}, 8 \mathrm{H},-\mathrm{CH}_{2}-\right), 2.22-$ 2.24 (t, J6.5 Hz, 4H, Cp- $\mathrm{CH}_{2}$ ), 3.50-3.54 (t, J6.3 Hz, 12H, OCH $), 3.96-3.97$ (d, 8H, Cp); ${ }^{3} \mathrm{CNMR}$ $\left(100 \mathrm{MHz}, \mathrm{CDCl}_{3}\right) \delta=-0.41\left(\mathrm{SiMe}_{2}\right), 12.8\left(-\mathrm{CH}_{3}\right), 15.1,18.1,27.6,29.9,30.2,33.8\left(\mathrm{CH}_{2}\right), 60.8$ $\left(\mathrm{OCH}_{2}\right), 66.7,67.8(\mathrm{Cp}), 88.3\left(\mathrm{C}_{1} \mathrm{Cp}\right)$; Anal. Calc. for $\mathrm{C}_{54} \mathrm{H}_{110} \mathrm{FeO}_{6} \mathrm{Si}_{6}: \mathrm{C}, 60.06 ; \mathrm{H}$ 10.27. Found: C, 59.85; H, 10.21\%.

1,1'-Bis[3-(tris(benzyloxydimethylsilyl)methyl)propyl]ferrocene (8e). Yellowish oil, FTIR $\left(\mathrm{KBr}, \mathrm{cm}^{-1}\right): v=3093,3030(\mathrm{Ar}-\mathrm{H}), 2929,2857(\mathrm{C}-\mathrm{H}), 1643,1496(\mathrm{C}=\mathrm{C}), 1253,838(\mathrm{Si}-\mathrm{C}), 1099$, 1027, 489 (Cp) 905 ( $\mathrm{Si}-\mathrm{O})$; ${ }^{1} \mathrm{HNMR}\left(400 \mathrm{MHz}, \mathrm{CDCl}_{3}\right) \delta=0.31$ (s, 36H, $\left.\mathrm{SiMe}_{2}\right), 1.25-1.28$ (m, $\left.8 \mathrm{H}, J 6 \mathrm{~Hz},-\mathrm{CH}_{2^{-}}\right), 2.22-2.26$ (t, 4H, J 7.2 Hz, Cp- $\left.\mathrm{CH}_{2}\right), 3.96-3.97$ (d, 8H, Cp), $4.68(\mathrm{~s}, 12 \mathrm{H}$, $\left.\mathrm{OCH}_{2}\right), 7.25-7.35$ (m, 8H, Ar- $H$ ), 7.33-7.34 (m, 24H, Ar- $H$ ); ${ }^{13} \mathrm{CNMR}\left(100 \mathrm{MHz}, \mathrm{CDCl}_{3}\right) \delta=-0.1$ ( $\left.\mathrm{SiMe}_{2}\right), 15.8,27.5,29.7,29.9,63.5\left(\mathrm{OCH}_{2}\right), 66.6,67.7(\mathrm{Cp}), 88.3\left(\mathrm{C}_{1} \mathrm{Cp}\right), 125.5,127.5,127.0$, 140.2 (Ar); Anal. Calc. for $\mathrm{C}_{66} \mathrm{H}_{98} \mathrm{FeO}_{6} \mathrm{Si}_{6}$ : C, 67.75; H 7.84, Found: C, 67.62; H, 7.79\%.

1,1'-Bis[4-(tris(methoxydimethylsilyl)methyl)butyl]ferrocene (8g). Yellowish oil, FTIR (KBr, cm-1): $v=3085(\mathrm{Cp}-\mathrm{H}), 2934,2829(\mathrm{C}-\mathrm{H}), 1649,1462(\mathrm{C}=\mathrm{C}), 1252,854,(\mathrm{Si}-\mathrm{C}), 1088,497(\mathrm{Cp})$ $982(\mathrm{Si}-\mathrm{O}) ;{ }^{1} \mathrm{HNMR}\left(400 \mathrm{MHz}, \mathrm{CDCl}_{3}\right) \delta=0.18$ (s, 36H, $\left.\mathrm{SiMe}_{2}\right), 1.41-1.46$ (m, 4H, $-\mathrm{CH}_{2}$ ), $1.50-$ $1.58\left(\mathrm{~m}, 4 \mathrm{H},-\mathrm{CH}_{2^{-}}\right), 1.68-1.72\left(\mathrm{t}, 4 \mathrm{H},-\mathrm{CH}_{2}-\right), 2.28-2.32\left(\mathrm{t}, 4 \mathrm{H}, J 7.6 \mathrm{~Hz}, \mathrm{CpCH}_{2}\right), 3.36(\mathrm{~s}, 18 \mathrm{H}$, $\left.\mathrm{OCH}_{3}\right)$, 3.94-3.95 (d, 8H, Cp); ${ }^{13} \mathrm{CNMR}\left(100 \mathrm{MHz}, \mathrm{CDCl}_{3}\right) \delta=-0.9\left(\mathrm{SiMe}_{2}\right), 16.0[-$ 
$\left.\mathrm{C}\left(\mathrm{SiM}_{2} \mathrm{OCH}_{3}\right)\right], 27.3,28.5,29.4,31.7\left(-\mathrm{CH}_{2}-\right), 48.9\left(\mathrm{OCH}_{3}\right), 66.7,67.6(\mathrm{Cp}), 87.5\left(\mathrm{C}_{1} \mathrm{Cp}\right)$; Anal. Calc. for $\mathrm{C}_{38} \mathrm{H}_{78} \mathrm{FeO}_{6} \mathrm{Si}_{6}$ : C, 53.36; $\mathrm{H}$ 9.19. Found: C, 53.25; H, 9.12\%.

1,1'-Bis[4-(tris(ethoxydimethylsilyl)methyl)butyl]ferrocene (8h). Yellowish oil, FTIR (KBr, $\left.\mathrm{cm}^{-1}\right): v=3085(\mathrm{Cp}-\mathrm{H}), 2969,2898(\mathrm{C}-\mathrm{H}), 1648,1442(\mathrm{C}=\mathrm{C}), 1251,849$ (Si-C), 1108, 1078, 498 (Cp), 943 (Si-O); ${ }^{1} \mathrm{HNMR}\left(400 \mathrm{MHz}, \mathrm{CDCl}_{3}\right) \delta=0.18$ (s, 36H, $\mathrm{SiMe}_{2}$ ), 1.12-1.15 (t, 18H, J=6.9, $\left.-\mathrm{CH}_{3}\right), 1.40-1.44$ (t, $\left.J 7.1 \mathrm{~Hz}, 4 \mathrm{H},-\mathrm{CH}_{2}-\right), 1.56-1.59$ (m, 4H, $\left.-\mathrm{CH}_{2}-\right), 1.70-1.74$ (t, $J 6.2 \mathrm{~Hz}, 4 \mathrm{H}$, $\mathrm{CH}_{2}$ ) , 2.27-2.31 (t, J 7.2 Hz, 4H, Cp- $\mathrm{CH}_{2}$ ), 3.57-3.62 (q, $\left.12 \mathrm{H}, J 6.9 \mathrm{~Hz}, \mathrm{OCH}_{2}\right), 3.95$ (s, 8H, Cp); ${ }^{13} \mathrm{CNMR}\left(100 \mathrm{MHz}, \mathrm{CDCl}_{3}\right) \delta=-0.35\left(\mathrm{SiMe}_{2}\right), 15.4\left(-\mathrm{CH}_{3}\right), 17.5,27.5,28.3,29.1,31.9\left(-\mathrm{CH}_{2}-\right)$, $56.6\left(\mathrm{OCH}_{2}\right), 66.6,67.5(\mathrm{Cp}), 88.3\left(\mathrm{C}_{1} \mathrm{Cp}\right)$; Anal. Calc. for $\mathrm{C}_{44} \mathrm{H}_{90} \mathrm{FeO}_{6} \mathrm{Si}_{6}: \mathrm{C}, 56.25 ; \mathrm{H}$ 9.66. Found: C, 56.12; H, 9.59\%.

1,1'-Bis[4-(tris(propoxydimethylsilyl)methyl)butyl]ferrocene (8i). Yellowish oil, FTIR (KBr, $\left.\mathrm{cm}^{-1}\right): v=3094(\mathrm{Cp}-\mathrm{H}), 2929(\mathrm{C}-\mathrm{H}), 1632,1461(\mathrm{C}=\mathrm{C}), 1252,833(\mathrm{Si}-\mathrm{C}), 1096,1027,488$ (SiC) $924(\mathrm{Si}-\mathrm{O}) ;{ }^{1} \mathrm{HNMR}\left(400 \mathrm{MHz}, \mathrm{CDCl}_{3}\right) \delta=0.19$ (s, 36H, SiMe 2$), 0.87-0.90$ (t, 18H, J 7.3 Hz, $\left.-\mathrm{CH}_{3}\right), 1.40-1.44,\left(\mathrm{t}, 4 \mathrm{H}, \mathrm{J} 7.2 \mathrm{~Hz},-\mathrm{CH}_{2}-\right), 1.51-1.52\left(\mathrm{~m}, 12 \mathrm{H},-\mathrm{CH}_{2}-\right), 1.53-1.55\left(\mathrm{~m}, 4 \mathrm{H},-\mathrm{CH}_{2}-\right)$, 1.70-1.74 (t, 4H, J 7.2 Hz, -CH $\left.2^{-}\right)$2.28-2.30 (t, 4H, J 7.6 Hz, Cp- $\left.\mathrm{CH}_{2}\right), 3.45-3.48$, (t, $12 \mathrm{H}, J 6.5$ $\left.\mathrm{Hz}, \mathrm{OCH}_{2}\right), 3.96(\mathrm{~s}, 8 \mathrm{H}, \mathrm{p}) ;{ }^{13} \mathrm{CNMR}\left(100 \mathrm{MHz}, \mathrm{CDCl}_{3}\right) \delta=-0.4\left(\mathrm{SiMe}_{2}\right), 9.5\left(-\mathrm{CH}_{3}\right), 15.3,24.7$, 27.5, 28.7, 29.3, 31.8, $62.8\left(\mathrm{OCH}_{2}\right), 66.6,67.7(\mathrm{Cp}), 87.7,\left(\mathrm{C}_{1} \mathrm{Cp}\right)$; Anal. Calc. for $\mathrm{C}_{50} \mathrm{H}_{102} \mathrm{FeO}_{6} \mathrm{Si}_{6}$ : C, 58.66; H 10.04 Found: C, 58.52; H, 9.91\%.

1,1'- Bis[4-(tris(butoxydimethylsilyl)methyl)butyl]ferrocene (8j). Yellowish oil, FTIR (KBr, $\left.\mathrm{cm}^{-1}\right): v=3096(\mathrm{Ar}-\mathrm{H}), 2925,2870(\mathrm{C}-\mathrm{H}), 1648,1463(\mathrm{C}=\mathrm{C}), 1252,830(\mathrm{Si}-\mathrm{C}), 1095,1034,484$ (Cp) 889 (Si-O); 488; ${ }^{1} \mathrm{HNMR}\left(400 \mathrm{MHz}, \mathrm{CDCl}_{3}\right): \delta=0.19$ (s, 36H, $\mathrm{SiMe}_{2}$ ), 0.88-0.91 (t, 18H, J= $\left.7.2,-\mathrm{CH}_{3}\right), 1.30-1.39\left(\mathrm{~m}, 12 \mathrm{H}, J 7 \mathrm{~Hz},-\mathrm{CH}_{2}-\right), 1.42-1.50\left(\mathrm{~m}, 16 \mathrm{H}, J 7.5 \mathrm{~Hz},-\mathrm{CH}_{2^{-}}\right), 1.56-1.60$ (t, $\left.4 \mathrm{H}, J 7.2 \mathrm{~Hz},-\mathrm{CH}_{2}-\right), 1.70-1.74$ (t, 4H, J 7.4 Hz, $\left.-\mathrm{CH}_{2}-\right), 2.29-2.33$ (t, $\left.4 \mathrm{H}, J 7.6 \mathrm{~Hz}, \mathrm{CpCH}_{2}\right), 3.48-$ $3.52\left(\mathrm{t}, 12 \mathrm{H}, J 6.4 \mathrm{~Hz}, \mathrm{OCH}_{2}\right), 3.95(\mathrm{~d}, 8 \mathrm{H}) ;{ }^{13} \mathrm{CNMR}\left(100 \mathrm{MHz}, \mathrm{CDCl}_{3}\right) \delta=-0.4\left(\mathrm{SiMe}_{2}\right), 12.8$, 15.1, 18.1, 27.6, 28.5, 28.7, 29.4, 31.7, 33.8, $60.9\left(\mathrm{OCH}_{2}\right), 66.7,67.5(\mathrm{Cp}), 88.4\left(\mathrm{C}_{1} \mathrm{Cp}\right)$; Anal. Calc. for $\mathrm{C}_{56} \mathrm{H}_{114} \mathrm{FeO}_{6} \mathrm{Si}_{6}$ : C, 60.71; H, 10.37, Found: C, 60.59; H, 10.25\%.

1,1'-Bis[4-(tris(benzyloxydimethylsilyl)methyl)butyl]ferrocene (8k). Yellowish oil, FTIR $\left(\mathrm{KBr}, \mathrm{cm}^{-1}\right): v=3091,3035(\mathrm{Ar}-\mathrm{H}), 2929,2854(\mathrm{C}-\mathrm{H}), 1645,1489(\mathrm{C}=\mathrm{C}), 1254,858(\mathrm{Si}-\mathrm{C}), 1065$, 1024, 484 (Cp) 945 (Si-O); ${ }^{1} \mathrm{HNMR}\left(400 \mathrm{MHz}, \mathrm{CDCl}_{3}\right) \delta=0.31$ (s, 36H, $\left.\mathrm{SiMe}_{2}\right), 1.44-1.52$ (m, $\left.4 \mathrm{H}, J 7.2 \mathrm{~Hz},-\mathrm{CH}_{2}-\right), 1.68-1.75$ (m, 4H, $-\mathrm{CH}_{2}-$ ), 1.86-1.90 (t, 4H, J 6.8 Hz, $-\mathrm{CH}_{2^{-}}$), 2.28-2.32 (t, $2 \mathrm{H}, J 7.6 \mathrm{~Hz}, \mathrm{CpCH}_{2}$ ), 3.96-3.97 (d, 8H, Cp), 4.68 (s, 12H, OCH $), 7.26-7.35$ (m, 30H, Ar- $H$ ); ${ }^{13} \mathrm{CNMR}\left(100 \mathrm{MHz}, \mathrm{CDCl}_{3}\right) \delta=-0.1\left(\mathrm{SiMe}_{2}\right), 15.9,27.6,28.7,29.4,31.9,63.5\left(\mathrm{OCH}_{2}\right), 66.5$, $67.7(\mathrm{Cp}), 88.4\left(\mathrm{C}_{1} \mathrm{Cp}\right), 125.5,125.7,127.0,140.2$ (Ar); Anal. Calc. for $\mathrm{C}_{68} \mathrm{H}_{102} \mathrm{FeO}_{6} \mathrm{Si}_{6}$ : C, 67.75; H 7.84, Found: C, 67.62; H, 7.79\%.

\section{Acknowledgements}

Financial support of this work by the University of Tabriz is gratefully appreciated. 


\section{References}

1. Kim, C.; Son, S.; Kim, B. J. Organomet. Chem. 1999, 588, 1. http://dx.doi.org/10.1016/S0022-328X(99)00335-6

2. Newkome, G. R.; Moorefield, C. N.; Vögtle, F. Dendritic Molecules: Concepts, Synthesis, Perspectives, VCH: Weinheim, 1996. http://dx.doi.org/10.1002/9783527614875

3. Reetz, M. T.; Lohmer, G.; Schwickardi, R. Angew. Chem. Int. Ed. 1997, 36, 1526. http://dx.doi.org/10.1002/anie.199715261

4. Lorenz, K.; Hölter, D.; Stühn, B.; Mülhaupt, R.; Frey, H. Adv. Mater. 1996, 8, 414. http://dx.doi.org/10.1002/adma.19960080509

5. Zeng, F.; Zimmerman, S. C. Chem. Rev. 1997, 97, 1681. http://dx.doi.org/10.1021/cr9603892

6. Trollsas, M.; Hawker, C. J.; Remenar, J. F.; Hedrick, J. L.; Johansson, M.; Ihre, H.; Hult, A. J. Polym. Sci. A: Polym. Chem. 1998, 36, 2793. http://dx.doi.org/10.1002/(SICI)1099-0518(19981115)36:15<2793::AID-POLA16>3.0.CO;2-M

7. Gudat, D. Angew. Chem. Int. Ed. 1997, 36, 1951. http://dx.doi.org/10.1002/anie.199719511

8. Kim, C.; Jeong, Y.; Jung, I. J. Organomet. Chem. 1998, 570, 9. http://dx.doi.org/10.1016/S0022-328X(98)00794-3

9. Uchida, H.; Kabe, Y.; Yoshino, K.; Kawamata, A.; Tsumuraya, T.; Masamune, S. J. A Chem. Soc. 1990, 112, 7077. http://dx.doi.org/10.1021/ja00175a062

10. Seyferth, D.; Son, D. Y.; Rheingold, A. L.; Ostrander, R. L. Organometallics 1994, 13, 2682. http://dx.doi.org/10.1021/om00019a026

11. Lambert, J. B.; Pflug, J. D.; Denari, J. M. Organometallics 1996, 15, 615. http://dx.doi.org/10.1021/om9505672

12. Sekiguchi, A.; Nanjo, M.; Kabuto, C.; Sakurai, H. J. Am. Chem. Soc. 1995, 117, 4195. http://dx.doi.org/10.1021/ja00119a046

13. Mathias, L. J.; Caroters, T. W. J. Am. Chem. Soc. 1991, 113, 4043. http://dx.doi.org/10.1021/ja00010a084

14. Casado, C. M.; Cuadrado, I.; Mora'n, M.; Alonso, B.; Lobeto, F.; Losada, J. Organometallics 1995, 14, 2618. http://dx.doi.org/10.1021/om00006a003

15. Lorenz, K.; Mülhaupt, R.; Frey, H. Macromolecules 1995, 28, 6657. http://dx.doi.org/10.1021/ma00123a036

16. Lambert, J. B.; Pflug, J. D.; Stern, C. L. Angew. Chem. 1995, 107, 106-108; Angew. Chem. Int. Ed. Engl. 1995, 34, 98. http://dx.doi.org/10.1002/ange.19951070126

17. Turrin, C. O.; Chiffre, J.; de Montauzon, D.; Balavoine, G.; Manoury, E.; Caminade A. M.; Majoral, J. P. Organometallics 2002, 21, 1891. 
http://dx.doi.org/10.1021/om010956y

18. Hovestad, N. J.; Ford, A.; Jastrzebski, J. T. B. H.; van Koten, G. J. Org. Chem. 2000, 65, 6338. http://dx.doi.org/10.1021/jo991726k

19. De Groot, D.; Emmerink, P. G.; Couke, C.; Reek, J. N. H.; Kamer, P. C. J.; van Leeuwen, P. W. N. M. Inorg. Chem. Commun. 2000, 3, 711.

http://dx.doi.org/10.1016/S1387-7003(00)00168-4

20. Kim, C.; Kwark, K. J. Polym. Sci. A: Polym. Chem. 2002, 40, 976.

http://dx.doi.org/10.1002/pola.10180

21. Kim, C.; Park, J. J. Organomet. Chem. 2001, 629, 194.

http://dx.doi.org/10.1016/S0022-328X(01)00865-8

22. Kim, C.; Kim, H.; Park, K. J. Organomet. Chem. 2003, 667, 96.

http://dx.doi.org/10.1016/S0022-328X(02)02148-4

23. Alonso, B.; González, B.; Garcia, B.; Rez-Oliva, E. R.; Zamora, M.; Casado, C. M.; Cuadrado, I. J. Organomet. Chem. 2001, 637, 642.

http://dx.doi.org/10.1016/S0022-328X(01)01140-8

24. Tagni, A.; Hayashi, T. Ferrocenes: Homogenous Catalysis, Organic Synthesis, Materials Science, VCH, New York, 1995.

25. Kong, Y. K.; Kim, J.; Choi, S.; Choi, S. B. Tetrahedron Lett. 2007, 48, 2033. http://dx.doi.org/10.1016/j.tetlet.2007.01.009

26. Shanmugam, P.; Madhavan, S.; Selvakumar, K.; Vaithiyanathan, V.; Viswambhar, B. Tetrahedron Lett. 2009, 50, 2213.

http://dx.doi.org/10.1016/j.tetlet.2009.02.161

27. Arrayas, R. G.; Adrido, J.; Carretero, J. C. Angew. Chem. Int. Ed. 2006, 45, 7674. http://dx.doi.org/10.1002/anie.200602482

28. Thomas, J. C. Chem. Rev. 2003, 103, 3101. http://dx.doi.org/10.1021/cr000427o

29. Dai, L. X.; Tu, T.; You, S. L.; Deng, W. P.; Hou, X. L. Acc. Chem. Res. 2003, 36, 659. http://dx.doi.org/10.1021/ar020153m

30. Green, M. L. H.; Marder, S. R.; Thompson, M. E.; Bandy, J. A.; Bloor, D.; Kolinsky, P. V.; Jones, R. J. Nature 1987, 330, 360. http://dx.doi.org/10.1038/330360a0

31. Molina, P.; Tarraga, A.; Lopez, J. L.; Martinez, J. C. J. Organomet. Chem. 1999, 584, 147. http://dx.doi.org/10.1016/S0022-328X(99)00126-6

32. Molina, P.; Pastor, A.; Vilaplana, M. J.; Velasco, M. D.; Ram Arellano, M. C. Organometallics 1997, 16, 5836.

http://dx.doi.org/10.1021/om9706166

33. Cain, C. E.; Mashburn, T. A. J.; Hauser, C. R. J. Org. Chem. 1961, 26, 1030. http://dx.doi.org/10.1021/jo01063a012

34. Zasloff, M. Nature 2002, 415, 389.

http://dx.doi.org/10.1038/415389a

35. Schmitt, M. A.; Weisblum, B.; Gellman, S. H. J. Am. Chem. Soc. 2004, 126, 6848. 
http://dx.doi.org/10.1021/ja048546z

36. Fernandes-Lopes, S.; Kim, H. S.; Choi, E. C.; Delgado, M.; Granja, J. R.; Khasanov, A.; Kraehenbuehl, K.; Long, G.; Weinberger, D. A.; Wilcoxen, K. M.; Ghadiri, M. R. Nature 2001, $412,452$. http://dx.doi.org/10.1038/35086601

37. Itazaki, M.; Kitami, O.; Tanabe, M.; Nishihara, Y.; Osakada, K. J. Organomet. Chem. 2005, $690,3957$.

http://dx.doi.org/10.1016/j.jorganchem.2005.05.030

38. Manners, I. J. Polym. Sci., Part A: Polym. Chem. 2002, 40, 179. http://dx.doi.org/10.1002/pola.10069

39. Hudson, R. D. A. J. Organomet. Chem. 2001, 637-639, 47. http://dx.doi.org/10.1016/S0022-328X(01)01142-1

40. Whittall, I. R.; Mcdonagh, A. M.; Humphrey, M. G. Advances in Organometallic Chemistry; Elsevier, 1998; Vol. 42, pp 291.

41. Miller, J. S.; Epstein, A. J.; Reiff, W. M. Chem. Rev. 1996, 88, 201. http://dx.doi.org/10.1021/cr00083a010

42. Ramírez-Oliva, E.; Cuadrado, I.; Casado, C. M.; Losada, J.; Alonso, B. J. Organomet. Chem. 2006, 691, 1131.

http://dx.doi.org/10.1016/j.jorganchem.2005.11.032

43. Moran, M.; Casado, C. M.; Cuadrado, I.; Losada, J. Organometallics 1993, 12, 4327.

http://dx.doi.org/10.1021/om00035a018

44. Safa, K. D.; Sharifi, E.; Tofangdarzadeh, S.; Hassanpour, A. Phosphorus, Sulfur, Silicon 2010 , $185,2433$.

http://dx.doi.org/10.1080/10426501003685783

45. Safa, K. D.; Oskoei, Y. M. J. Organomet. Chem. 2010, 695, 26. http://dx.doi.org/10.1016/j.jorganchem.2009.09.021

46. Safa, K. D.; Tofangdarzadeh, S.; Hassanpour, A. J. Organomet. Chem. 2009, 694, 4107. http://dx.doi.org/10.1016/i.jorganchem.2009.08.018

47. Safa, K. D.; Abbasi, H.; Teimuri-Mofrad, R. J. Organomet. Chem. 2013, 740, 56. http://dx.doi.org/10.1016/j.jorganchem.2013.04.027

48. Graindorge, H.; Mondet, J. C.; Vincent, C. H. U.S. Patent 5550 267; 1996.

49. Willy, W. E.; McKean, D. R.; Garcia, B. A. Bull. Chem. Soc. Jpn. 1976, 49, 1989. http://dx.doi.org/10.1246/bcsj.49.1989

50. Gröbel, T.; Seebach, D. Chem. Ber. 1977, 110, 852. http://dx.doi.org/10.1002/cber.19771100307

51. Safa, K. D.; Oskoei, Y. M. Arkivoc 2010, (10), 1.

52. Stiubianu, G.; Cazacu, M.; Nicolescu, A.; Hamciuc, V.; Vlad, S. J. Polym. Res. 2010, 17, 837 http://dx.doi.org/10.1007/s10965-009-9375-7 